\title{
The usefulness of neutrophil/lymphocyte count ratio in the diagnosis and prognosis of bacterial sepsis - An old parameter with new implications
}

\author{
Alina Orfanu*, Cristina Popescu, Cătălin Tilişcan, Adrian Streinu-Cercel, \\ Victoria Aramă, Ştefan Sorin Aramă
}

"Prof. Dr. Matei Bals" National Institute for Infectious Diseases, Bucharest, Romania, "Carol Davila” University of Medicine and Pharmacy, Bucharest, Romania

\begin{abstract}
Aims: To analyze the diagnostic role of neutrophil/lymphocyte count ratio (NLCR) in sepsis and to evaluate its usefulness in appreciating the prognosis of septic patients. Methods : A prospective analysis of patients diagnosed with sepsis between October 2015 and July 2017 was performed. A control group of patients with systemic inflammatory response syndrome (SIRS) with noninfectious causes was recruited. The sensitivity, specificity and AUC of NCLR for sepsis diagnosis were comparatively assessed with other biomarkers including fibrinogen (FIB), C-reactive protein (CRP) and procalcitonin (PCT). The baseline levels of NLCR were correlated with APACHE IV, APS and SOFA scores of severity. Results : We enrolled 105 patients with sepsis and 37 patients with noninfectious SIRS. $N L C R$ had significantly higher levels in the first group $(p<0.05)$. For an optimal cut-off value of 8.18 , the sensitivity, specificity and AUC of NLCR for sepsis diagnosis were 70.5\%, 72.2\% and 0.766, respectively. A cut-off value of 12.0 led to moderate sensitivity (58.7\%) and moderate-to-high specificity (89\%). For patients with SOFA score over 2 points, the characteristics did not significantly improve. The correlations between baseline NCLR and the severity scores were not statistically significant. Conclusion : NLCR levels were significantly higher in sepsis group compared to noninfectious SIRS group. NLCR had moderate sensitivity and specificity regarding sepsis diagnosis. Baseline levels of NLCR did not correlate with the severity scores, so its usefulness in sepsis prognosis was limited. Keywors: sepsis, diagnosis, prognosis, neutrophil/lymphocyte count ratio, inflammatory biomarkers Received: 11 th July 2019; Accepted: $3^{\text {rd }}$ November 2019; Published: $18^{\text {th }}$ November 2019

\section{Introduction}

Sepsis is characterized by high morbidity and mortality worldwide (1). Confronted with a septic patient with high risk of death, it is mandatory to have a prompt attitude in order to establish

a rapid diagnosis and an efficient therapy. For these goals, clinicians need good instruments such as clinical and biological tests, biomarkers, scores or algorithms. A very common and accessible test is the complete blood count (CBC), which seems to play an important role in the
\end{abstract}

*Corresponding author: Alina Orfanu, "Prof. Dr. Matei Bals" National Institute for Infectious Diseases, Bucharest, Romania. E-mail: alina.lobodan@yahoo.com 
diagnosis or prognosis of sepsis through some parameters such as neutrophil/lymphocyte count ratio (NLCR), mean platelet volume (MPV) or red cell distribution width (RDW).

NLCR is an easily achievable indicator which has been analyzed in several studies in recent years $(2,3,4)$. Its utility was evaluated not only in bacterial infections, but also in other pathologies characterized by inflammatory processes with different causes, like cardiovascular conditions (5), autoimmune disorders (6) or malignancies (7). The increase of NLCR is an effect of the immune system activation that leads to the overflow of a high number of neutrophils at the site of inflammation. The other process responsible is the redistribution and even apoptosis of lymphocytes as an adaptive mechanism, which induces lymphopenia (8). A thorough research in this field is needed because it can lead to a wider use of this low-priced test, especially in clinics from developing countries.

The first objective of this paper was to determine the sensitivity, specificity and area under the receiver operating characteristic (ROC) curve (AUC) of NLCR for sepsis diagnosis, in comparison with other inflammatory biomarkers commonly assessed in septic patients. The second objective was to correlate NLCR levels at baseline with the disease severity appreciated at admission through various severity scores. We hypothesized that NLCR could be a useful instrument in the diagnosis and prognosis of septic patients.

\section{Material and methods}

\section{Design and ethics}

The current study was a prospective observational analysis of patients diagnosed with community-acquired sepsis in a tertiary-care department of infectious diseases from Bucharest, Romania. The study received the approval from the Institutional Review Board. All patients or legal guardians signed the informed consent before the inclusion, according to the latest version of World Medical Association Declaration of Helsinki. The enrollment was performed between October 2015 and July 2017 and included consecutive subjects diagnosed with sepsis. Considering that the study started before the implementation of the new definition of sepsis (Sepsis-3), published in 2016 (9), the inclusion criteria were based on the old definition (Sepsis-2) (10). Thus, patients who gathered more than two of the criteria for systemic inflammatory response syndrome (SIRS) were included: fever (over 38 degrees C) or hypothermia (under 36 degrees C), tachycardia (heart rate over 90/minute), tachypnea (respiratory rate over $20 /$ minute) or $\mathrm{PaCO}_{2}$ under $32 \mathrm{mmHg}$ and leukocytosis (over 12 000/ $\mu 1$ ) or leukopenia (under $4000 / \mu 1$ ) or immature cells over $10 \%$ (11). The evidence of infection was the other mandatory element for the diagnosis, which was established based on clinical evidence, diagnostic imaging, positive cultures from blood, urine, sputum, bronchoalveolar lavage, pleural, pericardial, peritoneal, articular effusion, pathological secretions, serological or polymerase chain reaction (PCR) tests. Patients under 18 year-old and pregnant women were not included.

A control group consisting of patients with noninfectious SIRS was also recruited during the same period. Patients with more than two SIRS criteria, but with no evidence of infection were included. All patients from the control group were previously known or newly diagnosed with other pathologies that explained the inflammatory response: hematologic disorders, rheumatic conditions, solid tumors, allergies, cardiovascular or skin diseases. The recruitment was performed in an infectious diseases department, where the patients were admitted with the diagnosis of fever of unknown origin. The investigations performed excluded an infectious cause for the inflammatory syndrome. Furthermore, in all 
cases the symptoms persisted under broad-spectrum antibiotics and were solved under corticosteroids or under specific therapies according to the condition. Patients with bacterial superinfection, HIV-positive subjects, patients under 18 year-old and pregnant women were not included into the control group.

For all patients we analyzed several data, including demographic parameters (age, gender), predictors factors of severity (the number and characteristics of SIRS criteria, the presence of organic dysfunctions or septic metastases), primary sites of infection, etiological agents, biological tests at admission (CBC, including NLCR, MPV and RDW, fibrinogen - FIB, C-reactive protein - CRP, procalcitonin - PCT), in addition to the other tests and investigations usually performed in these cases (biochemistry, urinalysis, pulmonary X-ray, electrocardiogram etc).

The AUC, the sensitivity and specificity of each biomarker of inflammation for sepsis diagnosis were determined. The optimal cut-off value of each biomarker was identified. Even though the selection of patients was based on the old definition of sepsis (sepsis-2) (10), for more accurate results, these values were also calculated for septic patients with Sepsis-related Organ Failure Assessment (SOFA) score over two points (sepsis-3) (9).

For each patient with bacterial sepsis three scores of severity were calculated at admission: Acute Physiology and Chronic Health Evaluation (APACHE) IV, Admission Point Score (APS) and SOFA. The scores were subsequently correlated with the values of NLCR at baseline.

\section{Statistical analysis}

Normally distributed variables were expressed as mean \pm standard deviation (SD). For continuous variables with non-Gaussian distribution, the results were presented as median (interquartile range). In order to establish the variables distribution, Shapiro-Wilk and Kolmogorov-Smirnov tests as well as the aspect of histograms and Q-Q plots were analyzed. Differences in means between sepsis group and control group were evaluated using independent samples T-test. Chisquared test was used to assess the differences in proportions and Mann-Whitney U test was performed to establish the statistical significance between non-parametric continuous variables. The significance level was set at $p<0.05$. The comparison of the diagnostic accuracy of the biomarkers was performed using ROC curves analyses by calculating AUC. Correlations between non-normally distributed variables were computed using Spearman's rank-order correlation. The analyses were performed using SPSS 21.0 (SPSS Inc., Chicago, IL, USA).

\section{Results}

One hundred-five consecutive patients diagnosed with community-acquired sepsis were enrolled, with a median age of $57(43 ; 68.5)$ years and M:F sex ratio of $1: 1.38$. Between them, $85.7 \%$ of patients had a personal history of other diseases, including cardiovascular diseases, diabetes, chronic kidney disease, hepatic cirrhosis etc. Regarding the number of SIRS criteria, more than half of patients $(51.4 \%)$ had 2 criteria, $43.8 \%$ of patients had 3 criteria and $4.8 \%$ of them had 4 criteria. The criteria for SIRS (body temperature, heart rate, respiratory rate and number of leucocytes) were assessed for all patients. The primary site of infection was identified in $95.24 \%$ of cases: respiratory $(42.85 \%)$, abdominal $(24.9 \%)$, urinary $(23.8 \%)$, cutaneous $(6.66 \%)$. The infectious agent responsible for sepsis was isolated in $34.28 \%$ of cases. The most frequently isolated were: E. coli (52.77\%), Klebsiella pneumoniae (13.88\%), Streptococcus pneumoniae (11.11\%), Staphylococcus aureus (8.33\%). The median values of NLCR, MPV, RDW, FIB, CRP and PCT were also calculated at admission (Table 2). For the first objective of the study, 37 patients 
with a median age of $53(26.5 ; 68.5)$ years diagnosed with noninfectious pathologies responsible for SIRS were included into the control group. The distribution of patients from the control group regarding the noninfectious disease responsible for SIRS is detailed in table 1.

The group of septic patients and the control group were comparatively analyzed in Table 2 . Whereas the median age was similar between groups, the difference regarding sex ratio was statistically significant $(\mathrm{p}=0.034)$. The criteria for SIRS were also assessed. The number of leukocytes

Table 1. Distribution of patients in the control group, according to the noninfectious disorder responsible for SIRS

\begin{tabular}{lc}
\hline Noninfectious conditions & $\mathbf{n}(\mathbf{\%})$ \\
\hline Autoimmune disorders & $10(27)$ \\
\hline Hematologic diseases & $10(27)$ \\
\hline Solid tumors & $8(21.6)$ \\
\hline Cardiovascular diseases & $2(5.4)$ \\
\hline Skin disorders & $2(5.4)$ \\
\hline Drug toxicity & $3(8.1)$ \\
\hline
\end{tabular}

was the only parameter with significantly higher values in the first group $(\mathrm{p}=0.001)$. In regard to the mentioned inflammatory biomarkers, NLCR, CRP and PCT also presented higher levels in the group of septic patients $(p<0.001, p=0.002$ and $\mathrm{p}<0.001$, respectively). MPV, RDW and FIB did not register important differences between groups $(\mathrm{p}=0.425, \mathrm{p}=0.222$ and $\mathrm{p}=0.058$, respectively).

The AUC, sensitivity and specificity of NLCR for the diagnosis of bacterial sepsis according to the old definition of sepsis were calculated, in comparison with the other biomarkers. The optimal cut-off value of NLCR was estimated at 8.18 , with a sensitivity of $70.5 \%$ and a specificity of $72.2 \%$. The ROC curve of NLCR was comparatively evaluated with the ROC curves of the other biomarkers of inflammation (Figure 1). The AUC of NLCR for sepsis diagnosis was 0.766 (95\% CI 0.671-0.860), superior to MPV, RDW, FIB and CRP. The AUC of PCT was evaluated at $0.813(95 \%$ CI $0.721-0.904)$.

The AUC of the parameters and also the sensi-

Table 2. Baseline characteristics of sepsis versus control group patients.

\begin{tabular}{|c|c|c|c|}
\hline Variable & Bacterial sepsis $(n=105)$ & Control group $(n=37)$ & p value \\
\hline Age $\left(\right.$ years) ${ }^{a}$ & $57(43 ; 68.5)$ & $53(26.5 ; 68.5)$ & 0.104 \\
\hline Male & $44(41.90)$ & $23(62.16)$ & $0.034^{*}$ \\
\hline $\begin{array}{l}\text { Body temperature } \\
\text { (degrees Celsius) }\end{array}$ & $38.41 \pm 0.96$ & $38.38 \pm 0.63$ & 0.847 \\
\hline Heart rate (/minute) & $101.53 \pm 14.64$ & $96.73 \pm 13.82$ & 0.084 \\
\hline Respiratory rate (/minute) & $21.02 \pm 3.27$ & $20.38 \pm 1.72$ & 0.259 \\
\hline $\begin{array}{l}\text { Number of leukocytes } \\
(/ \mu 1)^{a}\end{array}$ & $\begin{array}{c}14510 \\
(10020 ; 20315)\end{array}$ & $\begin{array}{c}10350 \\
(5575 ; 13565)\end{array}$ & $0.001^{*}$ \\
\hline NLCR $^{a}$ & $13.01(6.27 ; 22.9)$ & $4.08(2.95 ; 8.47)$ & $<0.001^{*}$ \\
\hline $\operatorname{MPV}(f l)^{a}$ & $8.1(7.5 ; 8.7)$ & $7.9(7.33 ; 8.9)$ & 0.425 \\
\hline RDW (\%) ${ }^{a}$ & $13.8(13 ; 14.7)$ & $14(13.50 ; 15.22)$ & 0.222 \\
\hline FIB $(\mathrm{mg} / \mathrm{dl})^{a}$ & $635(474 ; 821)$ & $465(378.5 ; 780.5)$ & 0.058 \\
\hline $\operatorname{CRP}(\mathrm{mg} / \mathrm{l})^{\mathrm{a}}$ & $159(94.73 ; 213)$ & $89(21.73 ; 178.75)$ & $0.002^{*}$ \\
\hline PCT (ng/dl) ${ }^{a}$ & $2.33(0.35 ; 12.03)$ & $0.13(0.05 ; 0.22)$ & $<0.001^{*}$ \\
\hline
\end{tabular}

Results are expressed as mean \pm SD or number (\%). ${ }^{\text {a }}$ Data presented as median (IQR). * Statistical significance between groups $(\mathrm{p}<0.05)$. $\mathrm{p}$ values were obtained using independent samples T-test for continuous variables and Chi-squared or Mann-Whitney $\mathrm{U}$ test for variables with non-Gaussian distribution. NLCR, neutrophil/lymphocyte count ratio; MPV, mean platelet volume; RDW, red cell distribution width; FIB, fibrinogen; CRP, C-reactive protein; PCT, procalcitonin. 


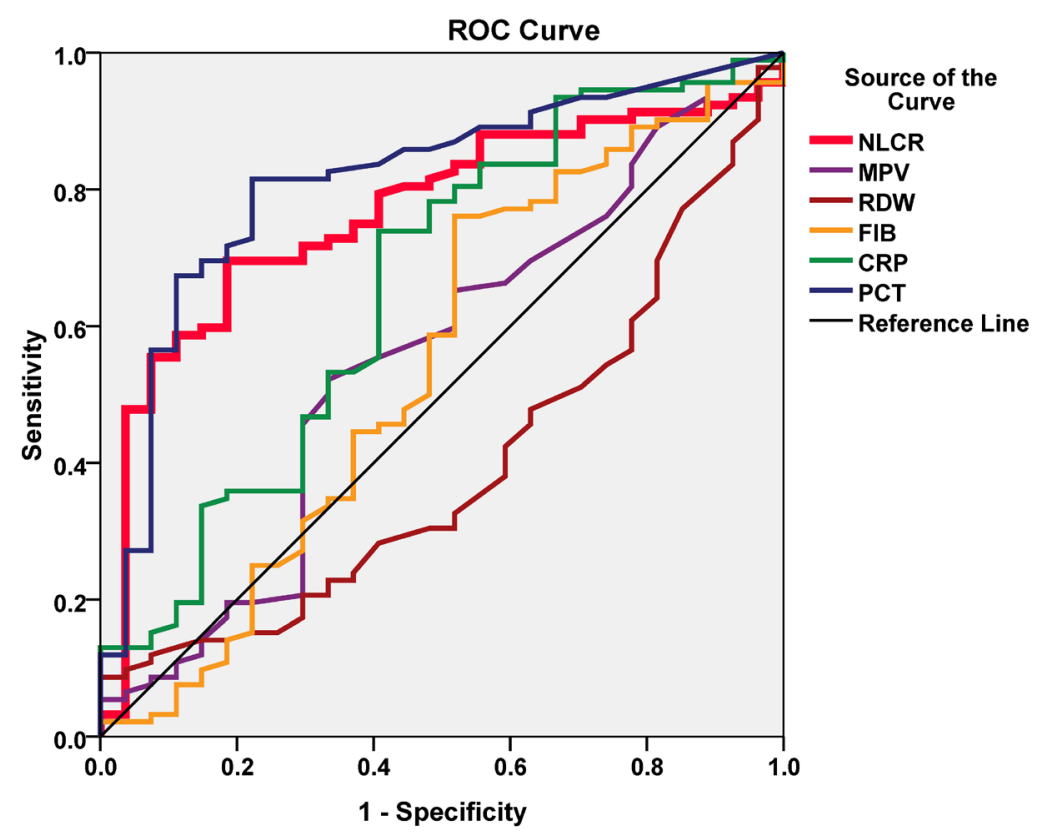

Fig. 1. ROC curves of NLCR, MPV, RDW, FIB, CRP and PCT for sepsis-2 diagnosis.

NLCR, neutrophil/lymphocyte count ratio; MPV, mean platelet volume; RDW, red cell distribution width; FIB, fibrinogen; CRP, C-reactive protein; PCT, procalcitonin.

tivity and specificity of the optimal cut-off values of each biomarker are illustrated in Table 3. According to our data, a randomly selected cutoff value of NLCR of 3.0 is correlated with high sensitivity (90.2\%) and low specificity (22.2\%) for sepsis diagnosis. A cut-off value of NLCR established at 12.0 leads to moderate sensitivity (58.7\%) and high specificity (89\%). For a more precise assessment, the sepsis group was subsequently divided in two categories, according to SOFA score: first group with SOFA score under 2 points -61 patients and the second one, with SOFA score $\geq 2$ points -44 patients. The AUC, sensitivity and specificity for sepsis diagnosis were calculated for the second group. The same control group was used. In this case, the AUC of NLCR for sepsis diagnosis was estimated at 0.766 (95\% CI 0.671-0.860), superior to MPV, RDW, FIB and CRP. The AUC of PCT was calculated at 0.911 (95\% CI 0.833-0.990). ROC curves of the mentioned biomarkers are presented in Figure 2.
For the second objective of the study, several predictors of severity were evaluated in the group of patients diagnosed with bacterial sepsis. Among them, $10(9.52 \%)$ presented septic metastases (cerebral, pulmonary, cutaneous or articular) and $70(66.66 \%)$ of them had at least one organ dysfunction (hematological, respiratory, renal, cardiovascular or hepatic). Moreover, three scores of severity were calculated at admission for each patient. The median of APACHE IV score was 28 $(20 ; 39.5)$, the median of APS was $18(13 ; 27)$, while the median of SOFA score was $1(0 ; 3)$.

In order to appreciate NLCR relationship with the prognosis of septic patients, NLCR levels at admission were correlated with the initial values of these scores. In comparison, we also evaluated the correlations between the initial levels of MPV, RDW, FIB, CRP and PCT and the severity scores (Table 4). For NLCR, FIB and CRP, these correlations were not statistically significant. NLCR value at admission did not correlate with APACHE IV (rho $=0.015$, 
Table 3. Characteristics of inflammatory biomarkers for sepsis diagnosis (sepsis-2 and sepsis-3, respectively)

\begin{tabular}{|c|c|c|c|c|c|c|c|c|}
\hline & \multicolumn{2}{|c|}{$\begin{array}{c}\text { AUC } \\
(95 \% \text { CI })\end{array}$} & \multicolumn{2}{|c|}{$\begin{array}{c}\text { Optimal } \\
\text { cut-off value }\end{array}$} & \multicolumn{2}{|c|}{$\begin{array}{c}\text { Sensitivity } \\
(\%)\end{array}$} & \multicolumn{2}{|c|}{$\begin{array}{c}\text { Specificity } \\
(\%)\end{array}$} \\
\hline & Sepsis-2 & Sepsis-3 & Sepsis-2 & Sepsis-3 & Sepsis-2 & Sepsis-3 & Sepsis-2 & Sepsis-3 \\
\hline NLCR & $\begin{array}{c}0.766 \\
(0.671-0.860)\end{array}$ & $\begin{array}{c}0.766 \\
(0.650-0.882)\end{array}$ & 8.18 & 7.87 & 70.5 & 70.7 & 72.2 & 70.4 \\
\hline MPV (fl) & $\begin{array}{c}0.554 \\
(0.425-0.682)\end{array}$ & $\begin{array}{c}0.579 \\
(0.440-0.717)\end{array}$ & 7.85 & 7.85 & 59.8 & 63.4 & 48.1 & 48.1 \\
\hline RDW (\%) & $\begin{array}{c}0.396 \\
(0.279-0.513)\end{array}$ & $\begin{array}{c}0.478 \\
(0.337-0.619)\end{array}$ & 13.95 & 13.95 & 42.4 & 53.7 & 40.7 & 40.7 \\
\hline FIB (mg/dl) & $\begin{array}{c}0.545 \\
(0.410-0.681)\end{array}$ & $\begin{array}{c}0.524 \\
(0.373-0.676)\end{array}$ & 551.5 & 473.5 & 58.7 & 78 & 51.9 & 44.4 \\
\hline CRP (mg/l) & $\begin{array}{c}0.658 \\
(0.534-0.781) \\
\end{array}$ & $\begin{array}{c}0.714 \\
(0.588-0.841) \\
\end{array}$ & 96.75 & 100.7 & 73.9 & 73.2 & 59.3 & 59.3 \\
\hline PCT (ng/dl) & $\begin{array}{c}0.813 \\
(0.721-0.904)\end{array}$ & $\begin{array}{c}0.911 \\
(0.833-0.990)\end{array}$ & 0.23 & 0.54 & 81.5 & 90.2 & 78.8 & 89.9 \\
\hline
\end{tabular}

AUC, area under the receiver operating characteristic curve; 95\% CI, 95\% confidence interval; NLCR, neutrophil/lymphocyte count ratio; MPV, mean platelet volume; RDW, red cell distribution width; FIB, fibrinogen; CRP, C-reactive protein; PCT, procalcitonin.

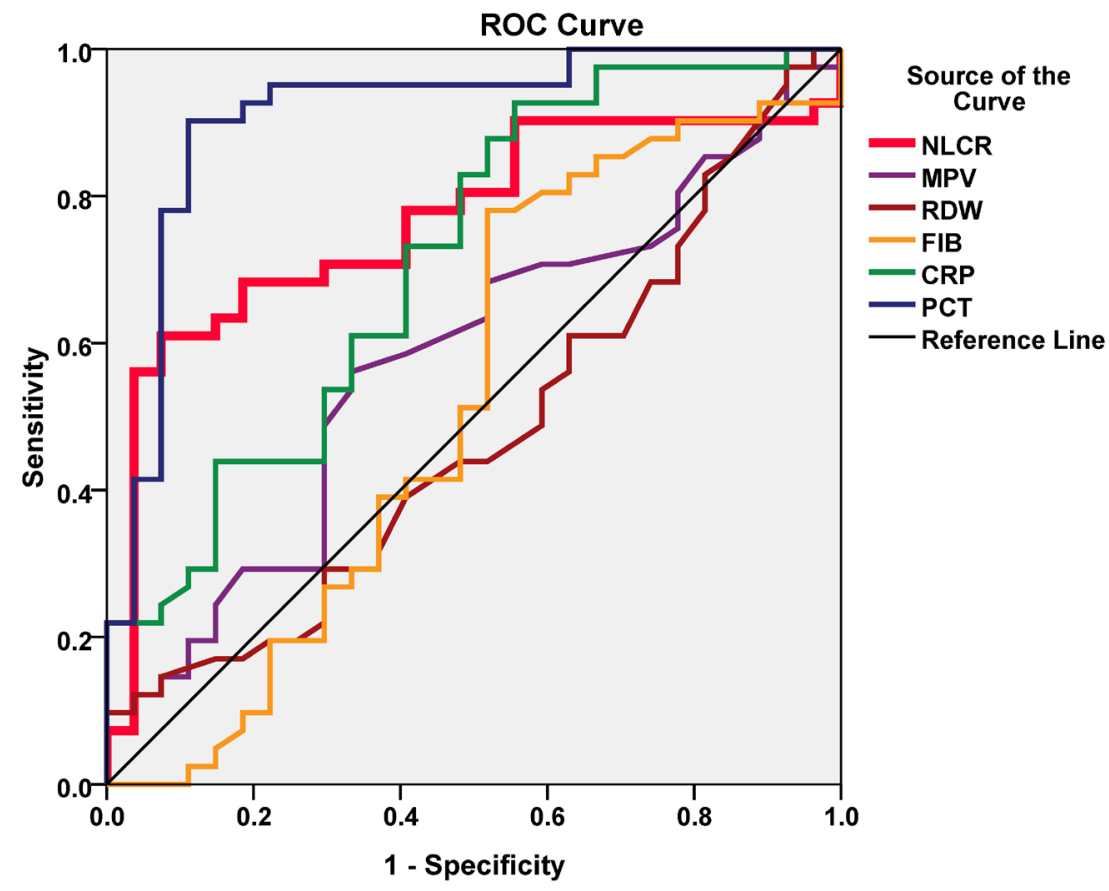

Fig. 2. ROC curves of NLCR, MPV, RDW, FIB, CRP and PCT for sepsis-3 diagnosis.

NLCR, neutrophil/lymphocyte count ratio; MPV, mean platelet volume; RDW, red cell distribution width; FIB, fibrinogen; CRP, C-reactive protein; PCT, procalcitonin. 
$\mathrm{p}=0.879)$, APS $(\mathrm{rho}=0.019, \mathrm{p}=0.844)$ or SOFA score ( $r h o=0.095, p=0.336$ ). On the other hand, MPV and RDW levels at baseline were correlated with SOFA score $(\mathrm{rho}=0.224, \mathrm{p}=0.022)$ and APACHE IV score $(\mathrm{rho}=0.245, \mathrm{p}=0.012)$ and SOFA score ( $r h o=0.301, p=0.002)$, respectively. PCT was the only parameter which was associated with the most reliable predictive value for sepsis prognosis. PCT levels at admission were correlated with all the mentioned severity scores (rho=0.305, $\mathrm{p}<0.001$, rho $=0.224, \mathrm{p}=0.011$ and rho $=0.528, \mathrm{p}<0.001$, respectively) and the correlations were statistically significant.

\section{Discussion}

The current study analyzed the usefulness of NLCR in establishing the diagnosis and evaluating the prognosis for septic patients. Regarding the diagnosis, NLCR levels were significantly higher in the group of sepsis compared to the controls. Furthermore, NLCR was characterized by good sensitivity, but limited specificity for sepsis diagnosis. Its specificity was moderate for the optimal cut-off value appreciated at 8.18. An increase of the cut-off value was associated with higher specificity. NLCR characteristics registered no significant improvement for patients with SOFA score over 2 points.

The role of NLCR in the diagnosis of sepsis represents the subject of several studies in the field, but the results are still questionable. Most of them also consider that this parameter is characterized by good sensitivity and low specificity for the diagnosis of sepsis. So far, Ljungstrom et al. demonstrated that a NLCR value of 3.0 is associated with a sensitivity of $95.1 \%(95 \%$ CI 93.3 - 96.9) and a specificity of $11.7 \%$ (95\% CI 9.7 - 13.7). Increasing this threshold at 10.0 changed the specificity to $60.8 \%$ (95\% CI 57.9 63.9) (12). In our study, for the same thresholds, NLCR specificity for sepsis diagnosis was higher $(22.2 \%$ for the cut-off value of 3.0 and $81.5 \%$ for the cut-off value of 12.0). Same authors concluded that the AUC of NLCR was 0.67 (95\% CI $0.64-0.69)$, inferior to the value obtained in our study $(0.766$ [95\% CI 0.671-0.860] for sepsis-2 criteria and 0.766 [95\% CI $0.650-0.882$ ] for sepsis-3 criteria).

Table 4. Correlations between the baseline levels of inflammatory biomarkers and the severity scores

\begin{tabular}{|c|c|c|c|c|c|}
\hline & \multirow{2}{*}{$\begin{array}{c}\text { Normal } \\
\text { values }\end{array}$} & \multirow{2}{*}{$\begin{array}{c}\text { Median } \\
\text { (IQR) }\end{array}$} & \multicolumn{3}{|c|}{ Correlations } \\
\hline & & & APACHE IV & APS & SOFA \\
\hline \multirow{2}{*}{ NLCR } & \multirow{2}{*}{-} & 13.01 & $\mathrm{rho}=0.015$ & $\mathrm{rho}=0.019$ & rho $=0.095$ \\
\hline & & $(6.27 ; 22.9)$ & $\mathrm{p}=0.879$ & $\mathrm{p}=0.844$ & $\mathrm{p}=0.336$ \\
\hline \multirow{2}{*}{ MPV (fl) } & \multirow{2}{*}{$7.8-11$} & 8.1 & rho $=0.060$ & rho $=-0.003$ & $\mathrm{rho}=0.224$ \\
\hline & & $(7.5 ; 8.7)$ & $\mathrm{p}=0.542$ & $\mathrm{p}=0.977$ & $\mathrm{p}=0.022 *$ \\
\hline \multirow{2}{*}{ RDW (\%) } & \multirow{2}{*}{$11.6-13.7$} & 13.8 & rho $=0.245$ & rho $=0.106$ & rho $=0.301$ \\
\hline & & $(13 ; 14.7)$ & $\mathrm{p}=0.012^{*}$ & $\mathrm{p}=0.282$ & $\mathrm{p}=0.002 *$ \\
\hline \multirow{2}{*}{ FIB (mg/dl) } & \multirow{2}{*}{$200-400$} & 635 & rho $=-0.133$ & rho $=-0.099$ & $\mathrm{r}=-0.172$ \\
\hline & & $(474 ; 821)$ & $\mathrm{p}=0.177$ & $\mathrm{p}=0.313$ & $\mathrm{p}=0.079$ \\
\hline \multirow{2}{*}{ CRP (mg/l) } & \multirow{2}{*}{$<3$} & 159 & $\mathrm{rho}=-0.038$ & rho $=0.057$ & $\mathrm{rho}=0.149$ \\
\hline & & $(94.73 ; 213)$ & $\mathrm{p}=0.713$ & $\mathrm{p}=0.577$ & $\mathrm{p}=0.143$ \\
\hline \multirow{2}{*}{ PCT (ng/dl) } & \multirow{2}{*}{$<0.05$} & 2.33 & $\mathrm{rho}=0.309$ & $\mathrm{rho}=0.230$ & rho $=0.485$ \\
\hline & & $(0.35 ; 12.03)$ & $\mathrm{p}=0.002 *$ & $\mathrm{p}=0.022 *$ & $\mathrm{p}<0.001 *$ \\
\hline
\end{tabular}

Data are shown as median (interquartile range). * Statistical significance between groups ( $<<0.05$ ). rho, Spearman's rank correlation coefficient; NLCR, neutrophil/lymphocyte count ratio; MPV, mean platelet volume; RDW, red cell distribution width; FIB, fibrinogen; CRP, C-reactive protein; PCT, procalcitonin; APACHE, Acute Physiology and Chronic Health Evaluation; APS, Admission Point Score; SOFA, Sepsis-related Organ Failure Assessment. 
The efficacy of NLCR in the diagnosis of sepsis was also evaluated in comparison with other biomarkers of inflammation. Zhang et al. observed that NLCR was inferior to PCT regarding the accuracy $(71.92 \%$ versus $80.6 \%)$ for sepsis diagnosis, but superior to CRP $(66.5 \%)$, RDW $(61.2 \%)$ and platelet distribution width - PDW $(61.5 \%)(2)$. The AUC of NLCR was 0.718 (95\% CI $0.625-0.811)$, inferior to PCT (0.829 [95\% CI $0.754-0.905])$, but superior to CRP (0.666 [95\% CI $0.568-0.764])$, RDW (0.621 [95\% CI $0.520-0.722])$ and PDW (0.636 [95\% CI $0.537-0.741])$. Our study also revealed the superiority of NLCR over MPV, RDW, FIB and CRP and the inferiority to PCT.

Another issue concerning NLCR evaluated in the last years was its usefulness in predicting the outcome of septic patients. However, in our paper, NLCR levels at baseline did not significantly correlate with the severity scores.

The utility of NLCR in critically ill patients admitted in the intensive care units was evaluated for the first time in 2001 in a study performed on 90 subjects with malignancies, 28 of cases being complicated with sepsis or septic shock. In comparison with the non-sepsis group, patients with systemic infections registered important neutrophilia and lymphopenia, inducing high values of NLCR. The authors proposed the term "neutrophil-lymphocyte stress factor" for this parameter and encouraged its implementation in the management of critically ill patients (13).

The predictive role of NLCR in sepsis was also evaluated by Terradas et al, who considered that a level over 7.0 is associated with a high mortality rate and also an ascendant trend during the first days of hospitalization in patients with septic shock is correlated with an increased risk of death (14). A more recent study estimated a threshold value for NLCR at 10.0 in order to stratify the risk of unfavorable outcome. Patients with NLCR over 10.0 developed more organ dysfunctions in comparison with those with
NLCR under $10(p=0.032)$. Moreover, the initial values of NLCR were significantly correlated with APACHE IV and APS scores and with the estimated rate of mortality (15).

In order to evaluate the correlation between NLCR and SOFA, APACHE II and Simplified Acute Physiology (SAPS) II scores on admission, Velissaris et al. performed an observational analysis of 50 patients with sepsis of multiple origins. All the correlations were statistically significant; SOFA - $\mathrm{r}=0.497, \mathrm{p}<0.001$; APACHE II - $r=0.411, p=0.003$; SAPS II $-r=0.445, p=0.001$ (16).

Another cohort study which included more than 5000 patients between 2001 and 2008 pointed out that NLCR could be a good predictor for the disease severity in critically ill patients. Focusing on selected cases of sepsis, the study showed no statistical significance of the correlation between NLCR and the 28-day mortality. Salciccioli et al. concluded that NLCR could indicate the inflammatory response, but its correlation with bacterial sepsis was not demonstrated (17).

In comparison with other biomarkers of inflammation, NLCR has good sensitivity (88\%) and positive predictive value $(87.5 \%)$, but moderate specificity $(75 \%)$ and negative predictive value ( $75 \%$ ) for sepsis prognosis. NLCR is situated on the second place after PCT, but before lactate, CRP, number of neutrophils, number of lymphocytes and number of leukocytes (18). Our results showed that NLCR is inferior to MPV, RDW and $\mathrm{PCT}$, concerning the prognosis of sepsis.

The limitations of our study can be considered the small number of patients, especially in the control group. The recruitment of the controls was performed in a department of infectious diseases, which could explain the low number of patients fulfilling the inclusion criteria. An interdisciplinary approach with the support of departments of rheumatology, hematology or oncology can be useful in the future. Another issue is the recruitment according to the old definition of 
sepsis, which can be responsible for the possible inclusion of patients with infection, but not with sepsis or for the possible exclusion of patients with sepsis, but with less than two SIRS criteria. This fact was partially solved retrospectively, through the selection of patients with SOFA score $\geq 2$ points. In this case, the data could be altered by the reduced number of patients. Future studies should be based on the new definition of sepsis. Another limitation consists in the singular correlation of baseline levels of NLCR with the severity scores. Future research should also assess the dynamical pattern of NLCR during hospitalization, in order to establish its usefulness in monitoring the outcome of septic patients.

In conclusion, NLCR is an accessible instrument with certain usefulness in the management of sepsis. Whereas its importance in the prognosis of sepsis is limited, NLCR has significantly higher values in septic patients, compared to the control group. Moreover, NLCR is characterized by better properties than other commonly used biomarkers of inflammation such as FIB or CRP concerning the diagnosis of sepsis.

\footnotetext{
Abbreviations

APACHE - Acute Physiology and Chronic Health Evaluation

APS - Admission Point Score

AUC - area under the ROC curve

$\mathrm{CI}$ - confidence interval

$\mathrm{CRP}$ - C-reactive protein

$\mathrm{CBC}$ - complete blood count

FIB - fibrinogen

HIV - human immunodeficiency virus

IQR - interquartile range

MPV - mean platelet volume

NLCR - neutrophil/lymphocyte count ratio

PCR - polymerase chain reaction

PCT - procalcitonin
}

PDW - platelet distribution width

RDW - red cell distribution width

ROC curve - receiver operating characteristic curve

SAPS - Simplified Acute Physiology

SIRS - systemic inflammatory response syndrome

SOFA - Sepsis-related Organ Failure Assessment

SD - standard deviation

\section{Acknowledgements}

This work is part of "Carol Davila" University of Medicine and Pharmacy doctoral programme.

\section{Authors' Contributions}

$\mathrm{AO}$ (Conception and design; Acquisition of data; Analysis and Interpretation of data; Writing Manuscript; Final approval), CP (Conception and design; Acquisition of data; Analysis and Interpretation of data; Writing Manuscript; Final approval); CT (Conception and design; Acquisition of data; Analysis and Interpretation of data; Writing Manuscript; Final approval), ASC (Conception and design; Acquisition of data; Analysis and Interpretation of data; Writing Manuscript; Final approval), VA (Conception and design; Acquisition of data; Analysis and Interpretation of data; Writing Manuscript; Final approval), SSA (Conception and design; Acquisition of data; Analysis and Interpretation of data; Writing Manuscript; Final approval). All the authors had equally contributed in elaborating the paper.

\section{Conflict of Interest}

None to declare.

\section{References}

1. Hajj J, Blaine N, Salavaci J, Jacoby D. The „Centrality of Sepsis": A Review on Incidence, Mortality, and 
Cost of Care. Healthcare (Basel). 2018;6(3):90. DOI: 10.3390/healthcare6030090

2. Zhang HB, Chen J, Lan QF, Ma XJ, Zhang SY. Diagnostic values of red cell distribution width, platelet distribution width and neutrophil-lymphocyte count ratio for sepsis. Exp Ther Med. 2016;12(4):2215-9. DOI: $10.3892 / \mathrm{etm} .2016 .3583$

3. Kaushik R, Gupta M, Sharma M, Jash D, Jain N, Sinha $\mathrm{N}$, et al. Diagnostic and Prognostic Role of Neutrophil-to-Lymphocyte Ratio in Early and Late Phase of Sepsis. Indian J Crit Care Med. 2018;22(9):660-3. DOI: 10.4103/ijccm.IJCCM_59_18

4. Pantzaris ND, Platanaki C, Pierrako C, Karamouzos V, Velissaris D. Neutrophil-to-lymphocyte Ratio Relation to Sepsis Severity Scores and Inflammatory Biomarkers in Patients with Community-acquired Pneumonia: A Case Series. J Transl Int Med. 2018;6(1):43-6. DOI: 10.2478/jtim-2018-0009

5. Tamhane UU, Aneja S, Montgomery D, Rogers EK, Eagle KA, Gurm HS. Association between admission neutrophil to lymphocyte ratio and outcomes in patients with acute coronary syndrome. Am J Cardiol. 2008;102:653-7. DOI: 10.1016/j.amjcard.2008.05.006

6. Yang Z, Zhang Z, Lin F, Ren Y, Liu D, Zhong R, Liang Y. Comparisons of neutrophil-, monocyte-, eosinophil-, and basophil- lymphocyte ratios among various systemic autoimmune rheumatic diseases. APMIS. 2017;125(10):863-71. DOI: 10.1111/apm.12722

7. Templeton AJ, McNamara MG, Seruga B, Vera-Badillo FE, Aneja P, Oca-a A, et al. Prognostic Role of Neutrophil-to-Lymphocyte Ratio in Solid Tumors: A Systematic Review and Meta-Analysis. J Natl Cancer Inst 2014;106(6):124. DOI: 10.1093/jnci/dju124

8. Riché F, Gayat E, Barthélémy R, Le Dorze M, Matéo J, Payen D. Reversal of neutrophil-to-lymphocyte count ratio in early versus late death from septic shock. Crit Care. 2015;19:439. DOI: 10.1186/s13054-015-1144-x

9. Singer M, Deutschman CS, Seymour CW, Shankar-Hari M, Annane D, Bauer M, et al. The Third International Consensus Definitions for Sepsis and Septic Shock (Sepsis-3). JAMA. 2016;315(8):801-10. DOI: 10.1001/ jama.2016.0287

10. Levy MM, Fink MP, Marshall JC, Abraham E, Angus $\mathrm{D}$, Cook D, et al. $2001 \mathrm{SCCM} / \mathrm{ESICM} / \mathrm{ACCP} /$
ATS/SIS International Sepsis Definitions Conference. Crit Care Med. 2003;4:1250-6. DOI: 10.1097/01. CCM.0000050454.01978.3B

11. Bone RC, Balk RA, Cerra FB, Dellinger RP, Fein AM, Knaus WA, et al. American College of Chest Physicians/Society of Critical Care Medicine Consensus Conference: Definitions for sepsis and organ failure and guidelines for the use of innovative therapies in sepsis. Crit Care Med. 1992;20(6):864-74. DOI: 10.1097/00003246-199206000-00025

12. Ljungstrőm L, Pernestig AK, Jacobsson G, Andersson R, Usener B, Tilevik D. Diagnostic accuracy of procalcitonin, neutrophil-lymphocyte count ratio, C-reactive protein, and lactate in patients with suspected bacterial sepsis. PLoS ONE. 2017;12(7):e0181704. DOI: 10.1371/journal.pone.0181704

13. Zahorec R. Ratio of neutrophil to lymphocyte counts-rapid and simple parameter of systemic inflammation and stress in critically ill. Bratisl Lek Listy. 2001;102(1):5-14.

14. Terradas R, Grau S, Blanch J, Riu M, Saballs P, Castells X, et al. Eosinophil Count and Neutrophil-Lymphocyte Count Ratio as Prognostic Markers in Patients with Bacteremia: A Retrospective Cohort Study. PLoS ONE. 2012;7(8):e42860. DOI: 10.1371/journal. pone. 0042860

15. Orfanu A, Arama V, Arama SS, Leustean A, Catana R, Negru A, et al. The diagnostic and prognostic role of Neutrophil to Lymphocyte count ratio in sepsis. BMC Infect Dis. 2016;16(4):31-76.

16. Velissaris D, Pantzaris ND, Bountouris P, Gogos C. Correlation between neutrophil-to-lymphocyte ratio and severity scores in septic patients upon hospital admission. A series of 50 patients. Rom J Intern Med. 2018;56(3):153-7. DOI: 10.2478/rjim-2018-0005

17. Salciccioli JD, Marshall DC, Pimentel MA, Santos MD, Pollard T, Celi LA, et al. The association between the neutrophil-to-lymphocyte ratio and mortality in critical illness: an observational cohort study. Crit Care. 2015;19:13. DOI: 10.1186/s13054-014-0731-6

18. Okashah AS, El-Sawy MM, Beshay BN, Abd El-Raouf A. Ratio of Neutrophil to Lymphocyte counts as a simple marker for sepsis and severe sepsis in Intensive Care Unit. Res Opin Anesth Intensive Care. 2014;2:39-45. 